\title{
Phase-2 reentry in cardiac tissue: Role of the slow calcium pulse
}

\author{
Inma R. Cantalapiedra, ${ }^{1}$ Angelina Peñaranda, ${ }^{1}$ Blas Echebarria, ${ }^{1}$ and Jean Bragard ${ }^{2}$ \\ ${ }^{1}$ Departament de Física Aplicada, Universitat Politècnica de Catalunya, Av. Dr. Marañón 44-50, E-08028 Barcelona, Spain \\ ${ }^{2}$ Departamento de Física y Matemática Aplicada, Ed. "Los Castaños," Facultad de Ciencias, \\ Universidad de Navarra, 31080 Pamplona, Spain
}

(Received 23 December 2009; revised manuscript received 6 June 2010; published 13 July 2010)

\begin{abstract}
Phase-2 re-entry is thought to underlie many causes of idiopathic ventricular arrhythmias as, for instance, those occurring in Brugada syndrome. In this paper, we study under which circumstances a region of depolarized tissue can re-excite adjacent regions that exhibit shorter action potential duration (APD), eventually inducing reentry. For this purpose, we use a simplified ionic model that reproduces well the ventricular action potential. With the help of this model, we analyze the conditions that lead to very short action potentials (APs), as well as possible mechanisms for re-excitation in a cable. We then study the induction of re-entrant waves (spiral waves) in simulations of AP propagation in the heart ventricles. We show that re-excitation takes place via a slow pulse produced by calcium current that propagates into the region of short APs until it encounters excitable tissue. We calculate analytically the speed of the slow pulse, and also give an estimate of the minimal tissue size necessary for allowing reexcitation to take place.
\end{abstract}

DOI: 10.1103/PhysRevE.82.011907 PACS number(s): 87.19.Hh, 87.19.R - 05.45.-a, 89.75.-k

\section{INTRODUCTION}

Cardiac arrhythmias and sudden cardiac death are among the most common causes of death in the industrialized world and predictions by the World Health Organization (WHO) are pointing towards a more dramatic situation for the $21 \mathrm{st}$ century [1]. Despite decades of theoretical and experimental research on the mechanisms responsible of cardiac arrhythmias, the causes of lethal arrhythmias are still incompletely understood.

A normal heart pumps blood throughout the body by means of an electrically synchronized contraction. This contraction is initiated by a change in the transmembrane potential of cardiac cells, starting at the sinoatrial node, whose self-excitatory cells act as the pacemakers of the heart. Subsequently, the electric wave spreads along the atria and the ventricles, and its activity can be monitored in an electrocardiogram (ECG). During normal rhythm (sinusal rhythm), thus, the heart beats regularly. In some situations, however, failures in regular wave propagation can cause the formation of reentrant waves (also called rotors), creating regions where excitation propagates in closed loops, with a period two or three times faster than normal. This corresponds to monomorphic or polymorphic tachycardia, depending if there is a single or multiple sources of reentrant waves. Although very often tachycardia terminates spontaneously, it may in some occasions degenerate into ventricular fibrillation (VF). The latter being a life-threatening cardiac disorder, where rotors are created and destroyed continuously, and synchronous excitation of the heart is lost. In this situation, the ability of the heart to pump blood decreases dramatically, and death occurs in a few minutes, unless a successful defibrillatory shock is provided.

In patients with structurally normal hearts polymorphic ventricular tachycardia (VT) and ventricular fibrillation (VF) account for $4 \%$ to $12 \%$ of the total sudden deaths each year. The formation of rotors is often due to the existence of dispersion of action potential (AP) repolarization in a region of cardiac muscle, which provides the substrate for reentry via, for instance, conduction block under rapid pacing, or phase- 2 reexcitations. In this latter mechanism, the AP dome is lost at some regions of tissue, but not at others, that can therefore reexcitate the already repolarized tissue [2]. Often, the loss of dome results from the interplay between the fast sodium current $I_{\mathrm{Na}}$ responsible of the depolarization of the cell (phase 0 of the AP), and the transient outward current $I_{t o}$, that creates the notch of the action potential (phase 1) [3,4]. If, as a result of the competition of these two currents, the transmembrane voltage remains below the threshold value for activation of the L-type calcium current $I_{\mathrm{CaL}}$, then the dome of the AP is lost. This is particularly relevant in the right ventricle (RV), where $I_{t o}$ has been shown to be stronger [5]. Phase- 2 reentry due to regions with loss of dome has been observed in situations resembling ischemia [6], or under conditions of elevated extracellular calcium with rapid pacing [7]. Another prominent example is Brugada syndrome [8]. This is a genetic disease that produces well-recognized changes in the electrocardiogram, characterized by an elevation of the ST-segment terminating in a negative $\mathrm{T}$ wave in the right precordial leads. Since its discovery in 1992, Brugada syndrome has been linked to more than 90 different mutations [9], including genes affecting the $\mathrm{Na}^{+}$and $\mathrm{Ca}^{2+}$ channels $[10,11]$. Particularly, between $10 \%$ and $30 \%$ of the cases of Brugada syndrome are related to mutations in the SCN5A gene [12,13], which encodes the $\alpha$-subunit of the voltage-gated sodium channel Nav1.5 [14]. These mutations have been shown to result in loss of function of the sodium channel, giving rise to either reduced $\mathrm{Na}^{+}$channel conductance or a change in the voltage dependence and dynamics of $I_{\mathrm{Na}}$ activation, inactivation, or reactivation [15]. The exact mechanism linking this genetic defect to the genesis of reentrant waves is, as yet, not completely understood. The substrate for reentry most probably appears when the action potential dome is lost at some epicardial sites but not at others, thus resulting in dispersion of repolarization within the epicardium. The origin of this effect may lie in the heteroge- 
neous distribution of transient outward current $\left(I_{t o}\right)$ conductance, which is known to vary transmurally from epicardium to endocardium, but has also been observed to change along the epicardium, from apex to base, and from apex to septum $[16,17]$. In fact, an epicardial dispersion of repolarization leading to VT via phase- 2 reentry has been observed in isolated canine ventricular myocytes with an $I_{t o}$ activator [18]. This link has been recently reinforced by the discovery that mutations in a gene affecting the strength of $I_{t o}$ current are related to cases of Brugada Syndrome [19].

Theoretical studies show that an inhomogeneous distribution of $I_{t o}$ can give rise to very disparate values of action potential duration (APD) in neighboring cells, leading to reexcitation [20-22]. This takes place via phase 2 reentry $[7,15]$, where electrotonic currents from a depolarized region of tissue (during calcium current entrance, or phase- 2 of the AP) are enough to provoke the firing of an AP in an adjacent area. All together, this explanation of the arrhythmogenic substrate in Brugada syndrome is known as the "repolarization disorder hypothesis." Simulations with the Luo-Rudy dynamic (LRd) model in a cable with a discontinuity of transient outward current conductance, $g_{\text {to }}$, have shown that $I_{\mathrm{Na}}$ is never triggered at the exact same point of the fiber where the discontinuity is located [22]. At the discontinuity point, rather, the recovery of the dome in the region with lower $g_{t o}$ produces a gradient of transmembrane voltage. Spatially, this results in a front in voltage that propagates slowly into the region of high $g_{\text {to }}$, most probably due to the activation of the L-type calcium current $I_{\mathrm{CaL}}$. This proceeds until the front reaches a point where the sodium gates have had enough time to recover, producing an influx of $I_{\mathrm{Na}}$ and a fast depolarization pulse.

Motivated by these computational results, in this work we study in detail under which circumstances does phase-2 reentry occur. We perform the analysis using a simplified cardiac model [23] that allows us to gain further insight into the mechanisms of reexcitation. In the next section we describe this simplified ionic model and how the numerical simulations are performed. In Sec. III, we study the mechanism of reexcitation. We start analyzing the conditions under which the APs lose the dome. This knowledge is later used to select the parameters for the simulations in a cable. We show that reexcitation is due to the existence of a slow pulse that propagates into the region of short APs until it triggers the fast pulse, similarly to the observations previously done in a realistic cardiac model [22]. The results from the numerical simulations are compared with theoretical predictions based on an analytical calculation of the slow pulse. Finally, phase-2 reentry and its corresponding ECG is studied in simulations of AP propagation in a geometrical model of the rabbit's ventricles with a gradient of $I_{t o}$ conductance. The last two sections are devoted to the discussion and the conclusions.

\section{MODEL EQUATIONS FOR THE ACTION POTENTIAL PROPAGATION}

Simulations of the propagation of the action potential (AP) throughout the heart are based on the standard monodomain propagation model [24,25],

$$
\frac{\partial \tilde{V}}{\partial t}=\nabla \cdot(\mathbf{D} \nabla \tilde{V})-\frac{\tilde{I}_{i o n}}{C_{m}}+\frac{\tilde{I}_{\text {stim }}}{C_{m}},
$$

where $\nabla$ is the nabla operator, $\widetilde{V}$ is the membrane potential (with commonly used "cardiac" units: $\mathrm{mV}$ ), $C_{m}$ is the membrane capacitance $\left(\approx 1 \mu \mathrm{F} \mathrm{cm} \mathrm{cm}^{-2}\right), \mathbf{D}=\sigma /\left(\widetilde{S} C_{m}\right)$ is the anisotropic conductivity (sometimes called diffusion) tensor (units: $\mathrm{cm}^{2} \mathrm{~ms}^{-1}$ ), $\sigma$ is the electrical conductivity (units: $\mathrm{m} \Omega^{-1} \mathrm{~cm}^{-1}$ ) and $\widetilde{S} \equiv S / V$ is the cell surface to volume ratio (units: $\mathrm{cm}^{-1}$ ). For the three-dimensional simulations, one must also take into account the inhomogeneous and anisotropic behavior of the diffusion parameter [26]. The values for the longitudinal diffusion $D_{\|}=10^{-3} \mathrm{~cm}^{2} / \mathrm{ms}$ and transverse diffusion $D_{\perp}=6.75 \times 10^{-5} \mathrm{~cm}^{2} / \mathrm{ms}$ are taken from the paper by Aguel et al. [27] and reflect the fact that the conduction velocity is more than three times larger along the fibers than in their transverse plane (i.e., transverse isotropic diffusion tensor). The simulations in a one-dimensional cable are carried out by replacing the spatial derivative term $\nabla \cdot(\mathbf{D} \nabla \tilde{V})$ by its one-dimensional counterpart $D \partial_{x x}^{2} \tilde{V}$ in Eq. (1). In this case, the diffusion parameter is set to the value for longitudinal diffusion $D_{\|}$. We discretize Eq. (1) using a simple forward explicit Euler method with $d x=0.015 \mathrm{~cm}$ and $d t=0.01 \mathrm{~ms}$.

In the present paper, cardiac excitation and propagation are simulated using a simple five-variable model [23]. This is a modification of the three-variable ionic model proposed by Fenton and Karma in 1998 [28]. We decompose the total membrane current into four components, i.e., $\widetilde{I}_{i o n}=\widetilde{I}_{f i}+\widetilde{I}_{s i}$ $+\widetilde{I}_{s o}+\widetilde{I}_{t o}$, where the sum contains a fast inward current $\widetilde{I}_{f i}$ $\left(\mathrm{Na}^{+}\right.$current plus the fast part of the $\mathrm{Ca}^{2+}$ current, in realistic cardiac models); a slow inward current, $\widetilde{I}_{s i}$ (correspondingly, $\mathrm{Ca}^{2+}$ current); a slow outward time-independent current, $\widetilde{I}_{s o}$; and a fast transient outward current, $\widetilde{I}_{t o}$, these last two corresponding to the sum of several $\mathrm{K}^{+}$currents in detailed models. The fast transient outward current $\widetilde{I}_{t o}$ is the newly added current with respect to the previous three-variable model proposed by Fenton and Karma [28]. Note that it is important to take it into consideration if one is interested in describing the Brugada syndrome. Indeed, the $\widetilde{I}_{t o}$ current is responsible for counteracting the effect of the sodium entry into the myocytes. In the Brugada syndrome, due to the weakness of sodium fluxes, the entrance of $\widetilde{I}_{t o}$ is often enough to result in a loss of dome of the action potential.

The explicit expressions of these currents in terms of the activation and deactivation gates are given in Appendix A. In the following, in order to alleviate the mathematical notation, we will rewrite Eq. (1) in dimensionless form by defining the membrane dimensionless voltage $V=\left(\tilde{V}-\tilde{V}_{\text {res }}\right) / \Delta \tilde{V}$, which varies roughly between zero to 1.5 and where $\tilde{V}_{\text {res }}$ $=-83 \mathrm{mV}$ is the resting potential and $\Delta \tilde{V}=85.7 \mathrm{mV}$ is an arbitrary potential difference. We also define the scaled currents $I_{f i}=\widetilde{I}_{f i} /\left(C_{m} \Delta \tilde{V}\right)$ (and similar expressions for the others currents, $I_{s o} ; I_{t o} ; I_{s i}$, which have all dimension of inverse time 
a)

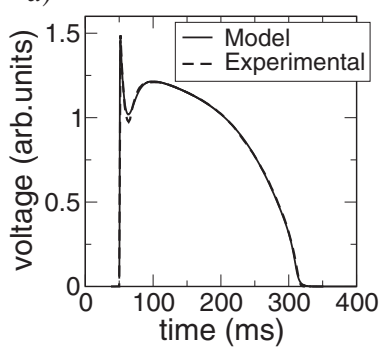

b)

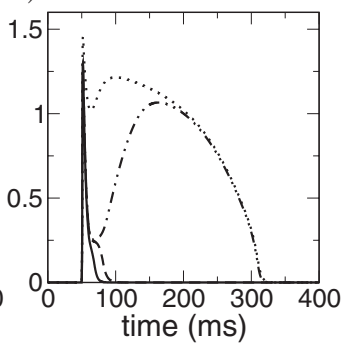

FIG. 1. (a) Comparison between the five-variable model and an experimental action potential in ventricular tissue, in a cell paced with a stimulation period of $1 \mathrm{~s}$. (b) Action potentials for the standard, or wild-type (WT), parameters (dotted line) and modified parameters showing a shortening of the APD obtained by increasing the transient outward current conductance $g_{t o}: g_{t o}=2.45 \times g_{\text {to }}^{W T}$ (dotdashed line); $g_{t o}=2.46 \times g_{t o}^{W T}$ (dashed line); $g_{t o}=2.56 \times g_{t o}^{W T}$ (solid line).

and units are $\mathrm{ms}^{-1}$ ). In Fig. 1(a) we compare the fit of the five-variable model with the experimental epicardial AP given in the Ref. [29]. The corresponding parameters are given in Appendix A (Table I). In the following we will refer to these parameters as wild type (WT). The fitting technique is based on a standard nonlinear optimization routine from Matlab based on the Levenberg-Marquardt algorithm [30] where one minimizes at each iteration step the mean quadratic error between the experimental data and the output of the model.

TABLE I. Values of the parameters in the model (shown with three significant digits). They are determined using a routine that fits the model to the experimental AP values in [29].

\begin{tabular}{lcc}
\hline \hline Parameters & Numerical values & Units \\
\hline$\tau_{h_{+}}$ & 39.2 & $\mathrm{~ms}$ \\
$\tau_{h_{-}}$ & 3.47 & $\mathrm{~ms}$ \\
$\tau_{f_{+}}$ & 40.0 & $\mathrm{~ms}$ \\
$\tau_{f_{-}}$ & 180 & $\mathrm{~ms}$ \\
$\tau_{r_{+}}$ & 9.13 & $\mathrm{~ms}$ \\
$\tau_{r_{-}}$ & 6.93 & $\mathrm{~ms}$ \\
$\tau_{s_{+}}$ & 39.2 & $\mathrm{~ms}$ \\
$\tau_{s_{-}}$ & 3.47 & $\mathrm{~ms}$ \\
$g_{f i}$ & 3.47 & $\mathrm{~ms}^{-1}$ \\
$V_{f i}$ & 1.60 & \\
$g_{s o}$ & 0.0240 & $\mathrm{~ms}^{-1}$ \\
$g_{s i}$ & 0.0529 & $\mathrm{~ms}^{-1}$ \\
$\beta_{1}$ & 9.89 & \\
$\beta_{2}$ & 4.44 & \\
$V_{1}$ & 0.250 & \\
$V_{2}$ & 1.26 & \\
$g_{t o}$ & 3.49 & \\
$V_{c}$ & 0.130 & \\
$V_{r}$ & 0.200 & \\
\hline \hline & & \\
\hline
\end{tabular}

\section{MECHANISMS FOR REEXCITATION IN CARDIAC TISSUE PRESENTING DISPERSION OF APD}

In Figure 1(b), we show that a large $I_{t o}$ conductance can drastically reduce the effect of the slow inward (calcium) current $I_{s i}$. In the Brugada syndrome this is exacerbated by a reduction of the depolarization current, caused by a rapid inactivation of the membrane sodium channels, leading to a decrease of the inward current at the initiation of the action potential. In the epicardium tissue, where the potassium mediated transient outward current is large, this may result in the disappearance of the characteristic dome of the action potential. If this loss is not homogeneous, dispersion of repolarization ensues. This is a strong proarrhythmic situation. Indeed, the region without dome becomes newly excitable by the neighboring regions that have not yet repolarized, creating the right ingredient for initiating phase 2 reentry and inducing tachycardia and fibrillation [31].

The origin of a heterogeneity in repolarization is not completely clear. It could be due to a gradient in $I_{t o}$, since it has been observed that the expression of the fast $\left(I_{t o f}\right)$ and slow $\left(I_{t o s}\right)$ components of this current change from apex to base, or from apex to septum $[16,34,35]$. In Brugada syndrome, it could also be due to a heterogeneous distribution in the strength of the mutation affecting the $I_{\mathrm{Na}}$ current, although, to our knowledge, there is no clear evidence of this point. Whichever is the source of heterogeneity, we claim that the mechanism for phase- 2 reentry will remain the same, as long as the L-type calcium current is not affected.

In the following, we will consider first the conditions for the loss of dome in a single cell. Then, study a cable with a gradient in the transient outward current conductance $g_{t o}$, and the ensuing dispersion of repolarization. We will show that this heterogeneity is almost independent of the strength of the gradient. We then consider a spatial variation in $g_{\text {to }}$ (and also in $\tau_{h_{-}}$) in the form of a Boltzmann gradient (as in Ref. [21]) and study the mechanism for reexcitation in this situation.

\section{A. Loss of dome and dispersion of repolarization}

\section{Loss of the AP dome in a single cell (OD simulations)}

The appearance or not of a dome depends on the value of the transmembrane potential at the end of the phase- 1 of the action potential. Therefore, three parameters are the most important: the strength $g_{f i}$ and the inactivation time $\tau_{h}$ of the fast inward current $I_{f i}$, and the strength of the transient outward current $g_{\text {to }}$. Another important parameter is related with the slow inward current $I_{s i}$ (or $I_{\mathrm{CaL}}$ in more realistic models), through its conductance $g_{s i}$. As shown in Fig. 2, the dome can be lost either by decreasing $g_{f i}$ or $\tau_{h_{-}}$for fixed $g_{t o}$, or by increasing $g_{t o}$ for fixed $g_{f i}$ or $\tau_{h_{-}}$. In any of these cases, the transition from dome to domeless AP is always very sharp. In contrast, the parameter $g_{s i}$ has a different effect. When $g_{s i}$ is low, the AP is short, and increases gradually as $g_{s i}$ is increased, with a narrow band of domeless APs at higher values of $g_{t o}$. For large values of $g_{s i}$ [see Fig. 2(f)], the region of short APDs disappears, and there is a direct transition from no AP to long APDs. 

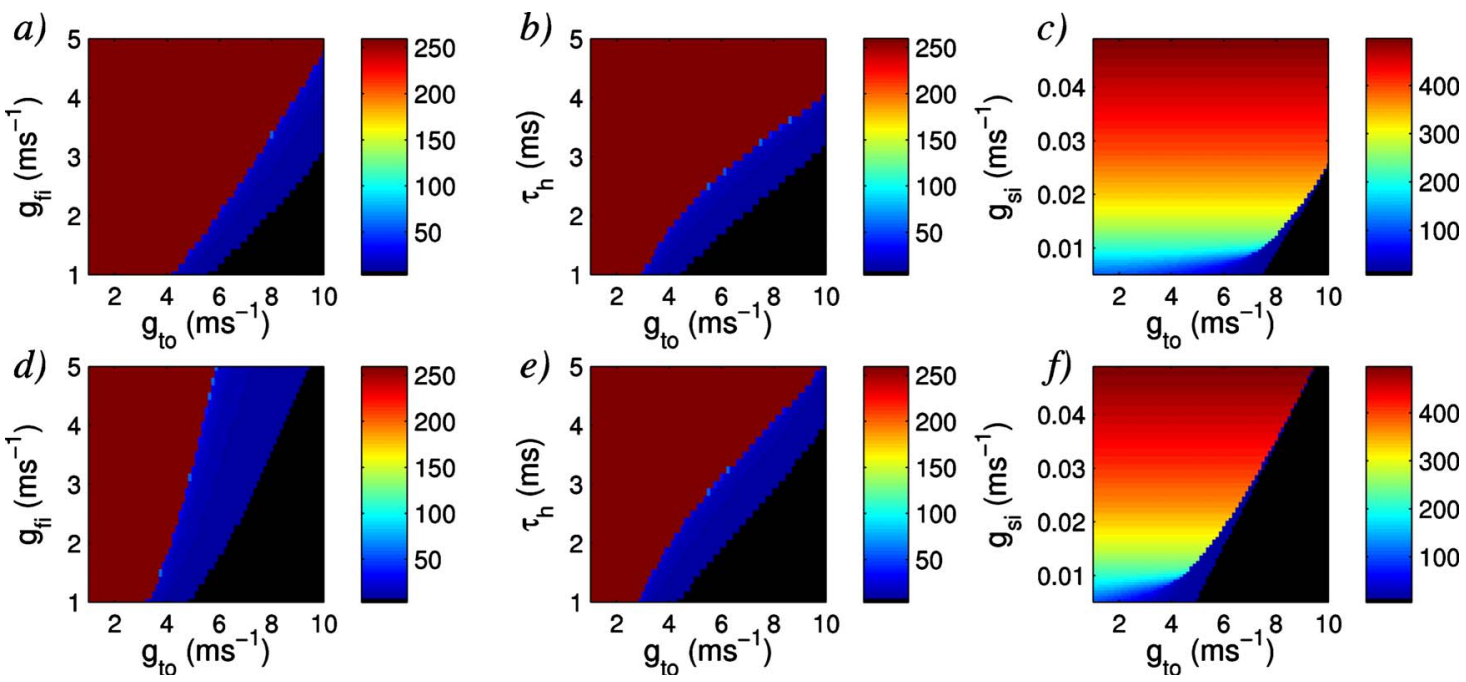

FIG. 2. (Color online) Values of the APD as a function of several parameters of our model. Black regions means no AP, blue intermediate regions depict domeless AP, and red regions correspond to normal AP. We show in the upper row (a, b, and c) the results for normal epicardium, while in the lower row (d, e, and f) we have introduced the following changes: (d) and (f) $\tau_{h_{-}}=2.31 \mathrm{~ms}=\tau_{h}^{W T} / 1.5$, (e) $g_{f i}=2.5 \mathrm{~ms}^{-1}=g_{f i}^{W T} / 1.39$.

\section{Dispersion of repolarization due to a gradient in $g_{\text {to }}$ (1D simulations)}

Optical mapping has been used to obtain maps of AP duration on the ventricles, emphasizing the relation between dispersion of repolarization and phase-2 reentry [31]. The underlying cause of this heterogeneous distribution of APD remains less clear. From the results of the previous section (Sec. III A 1), one expects that small changes of electrophysiological properties should be enough to provoke noticeable dispersion of repolarization. To clarify this point, we have performed simulations in a cable with a gradient of $g_{t o}$, and measured the resulting APD at all locations in the fiber. As can be seen in Fig. 3, there exists always a localized discontinuity in APD, that is furthermore almost independent of the strength of the gradient. This is in agreement with the results of Sec. III A 1 where simulations in single cell already showed that a minute change in $g_{t o}$ can result in a large variation of APD (see Fig. 2).

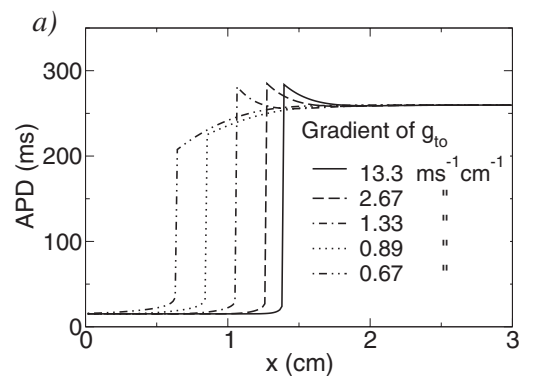

\section{B. Reexcitation in a cable}

\section{Reexcitation}

Up to this point, we have studied the conditions for the appearance of dispersion of repolarization. However, to obtain phase- 2 reentry, reexcitation of the repolarized region must occur. We now study this point in a cable paced at one end. We will consider changes of the electrophysiological properties modeled by a Boltzmann gradient of the type:

$$
\begin{gathered}
g_{t o}=g_{t o}^{\min }+\frac{g_{t o}^{\max }-g_{t o}^{\min }}{1+\exp [(x-L / 2) / s]}, \quad \text { or } \\
g_{t o}=g_{t o}^{\max }-\frac{g_{t o}^{\max }-g_{t o}^{\min }}{1+\exp [(x-L / 2) / s]}
\end{gathered}
$$

and equivalent expressions for other electrophysiological quantities. The parameter $s$ is related with the strength of the gradient. Unless otherwise stated we will take $s=0.05 \mathrm{~cm}$.

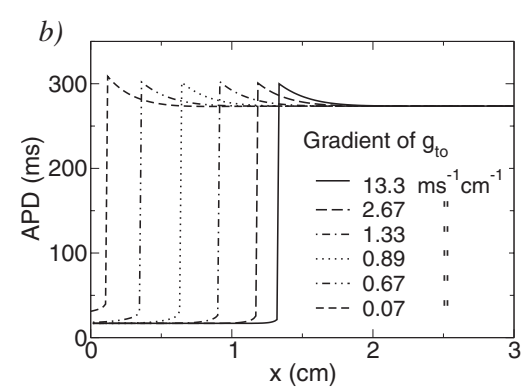

FIG. 3. Value of the APD at all spatial locations in a fiber of length $L=3 \mathrm{~cm}$. All points in the cable are stimulated at once. The spatial distribution of the parameter $g_{t o}$ follows a ramp given by $g_{t o}=4.5 \mathrm{~ms}^{-1}+G(L / 2-x)$. Here we show the influence of the gradient (slope) $G$. The ramp connects to the constant values: $g_{t o}=5.5 \mathrm{~ms}^{-1}$ at the left side of the cable and to $g_{t o}=3.5 \mathrm{~ms}^{-1}$ at the right side of the cable. Note that for the smallest gradient in (b) $\left(\mathrm{G}=0.07 \mathrm{~ms}^{-1} \mathrm{~cm}^{-1}\right)$, we have taken $g_{t o}=5.16 \mathrm{~ms}^{-1}+G(L / 2-x)$, so that it could fit in the same figure as with the other gradient parameter. The difference between (a) and (b) lies in the fact that in (a) we take $g_{s i}=g_{s i}^{W T}$, while in (b) $g_{s i}=1.08 \times g_{s i}^{W T}$. 

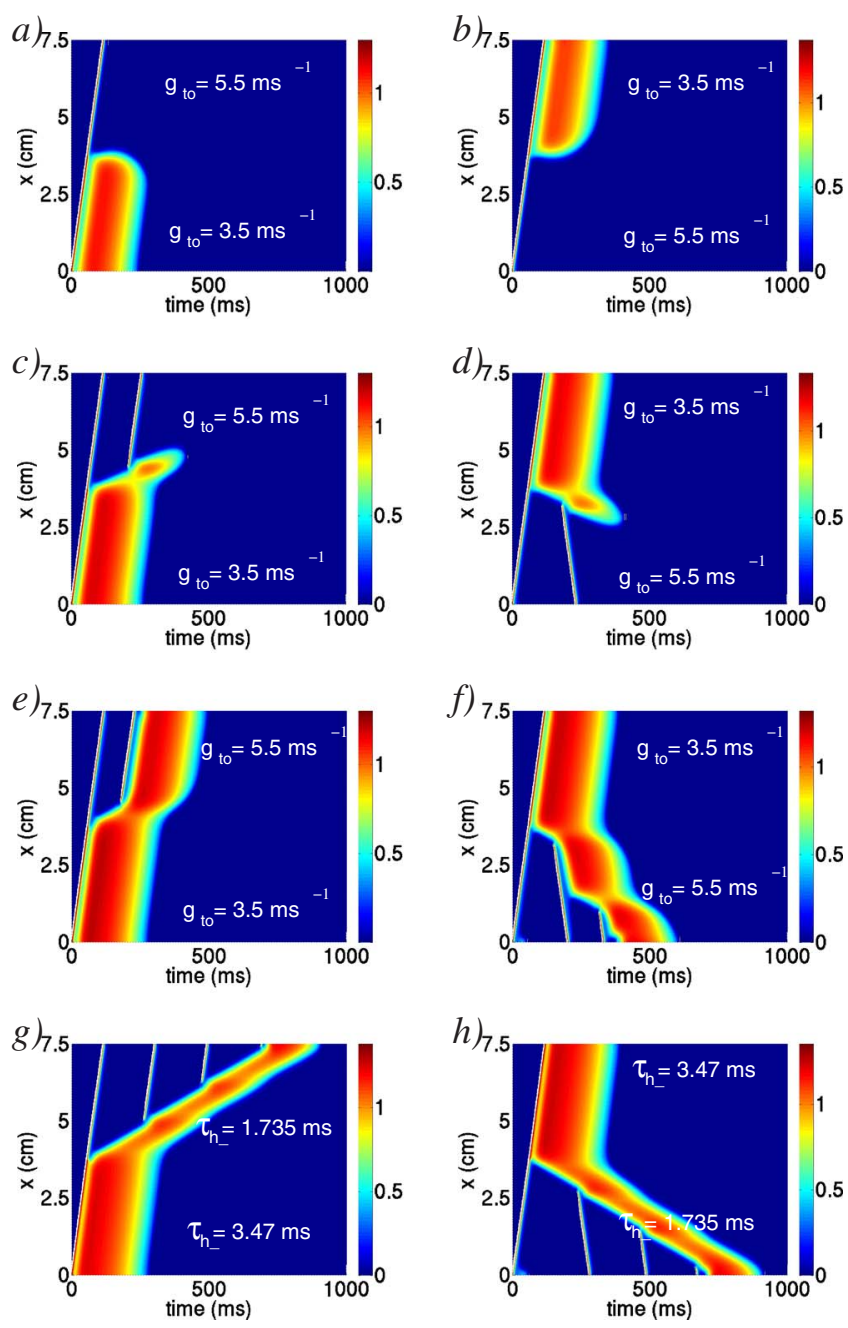

FIG. 4. (Color online) Space-time plots showing the evolution of a pulse in a $1 \mathrm{D}$ fiber with a gradient of $I_{t o}$ conductance, or $I_{f i}$ inactivation times. The length of the cable is $L=7.5 \mathrm{~cm}$, and the total simulation time is $1 \mathrm{~s}$. Depending on the conductance of the slow inward current $g_{s i}$ the system may present no reexcitation (a) and (b), or multiple reexcitations (FR or BR), with or without recovering the dome. The values of the parameters are for (a) and (b) $g_{s i}=0.9 \times g_{s i}^{W T}$; in (c) and (d), $g_{s i}=g_{s i}^{W T}$; in (e) and (f), $g_{s i}=1.08$ $\times g_{s i}^{W T}$. For (a) to (f), one sets $g_{\text {to }}^{\text {min }}=3.5$ and $g_{\text {to }}^{\max }=5.5 \mathrm{~ms}^{-1}$ in the two sides of the cable and $\tau_{h_{-}}=\tau_{h_{-}}^{W T} / 1.5$. In panels $(\mathrm{g})$ and $(\mathrm{h})$, one sets $g_{s i}=1.1 \times g_{s i}^{W T}$ and $g_{t o}=4.5 \mathrm{~ms}^{-1}$, with $\tau_{h_{-}}^{\max }=3.47$ and $\tau_{h_{-}}^{\min }=1.735 \mathrm{~ms}^{-1}$ in each side of the spatial domain.

The two expressions correspond to a gradient that decreases or increases with $x$. In Fig. 4 we illustrate several cases in which reexcitation (in the form of backward reexcitation BR or forward reexcitation FR) can be induced, for different values of the parameter $g_{s i}$. Dispersion of repolarization is obtained by applying a gradient in $g_{\text {to }}$ values to the fiber following the expression of the Eq. (2), except in the last panels [see Figs. 4(g) and 4(h)], where we rather set a change of $I_{f i}$ inactivation time $\tau_{h_{-}}$. Typically, as the pulse travels from the region with large (small) $g_{t o}$ to the region of small (large) $g_{\text {to }}$, it recovers (loses) the dome [Figs. 4(a) and 4(b)]. For larger values of $g_{s i}$ [Figs. 4(c) and 4(d)] the dome may

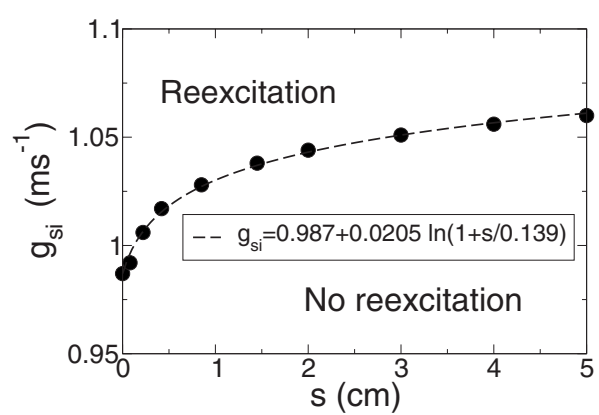

FIG. 5. Critical value of $g_{s i}$ necessary to obtain reexcitation as a function of the strength of the gradient in $g_{t o}$. We take $g_{t o}=3.5$ $+2 /(1+\exp [(x-L / 2) / s])$. In the simulations with a shallow gradient the length of the system is increased as to reach values of $g_{t o}$ within $1 \%$ of the maximum and minimum.

propagate back into the region of large $g_{t o}$, resulting in a delayed dome (an early afterdepolarization phenomenonEAD). Depending on the values of the $I_{s i}$ current, diffusion and inactivation time, this delayed dome can, in fact, produce reexcitation ( $I_{f i}$ entrance), as it is displayed in Figs. 4(c) and 4(d), or even multiple reexcitations, as shown in Figs. 4(e) and 4(f). Pacing from one end of the cable (here from the lower end), the reexcitation can be either antidromic [BR, see Figs. 4(d) and 4(f)] or orthodromic [FR, see Figs. 4(c) and 4(e)], depending on which side of the cable has the largest value of $g_{t o}$. The same situation happens when $g_{\text {to }}$ is constant along tissue, but the dispersion of APD is caused by a gradient in the fast inward current inactivation time $\tau_{h_{-}}$, as it could be the case in the Brugada syndrome [Figs. $4(\mathrm{~g})$ and $4(\mathrm{~h})]$.

\section{Effect of a change in the gradient}

In the previous subsection we have shown that, increasing $g_{s i}$ beyond a critical value, it is possible to obtain reexcitation when we impose a gradient of electrophysiological properties. Now we want to address the question of how dependent is the critical value of $g_{s i}$ on the strength of the gradient. For that, we have considered a pulse propagating in a gradient of decreasing values of $g_{t o}$, and calculated the critical value of $g_{s i}$ as a function of $s$ (Fig. 5). The resulting curve seems to fit very well by an expression of the form $g_{s i}=g_{s i}^{*}+a_{0} \ln (1$ $\left.+s / a_{1}\right)$, where $g_{s i}^{*}$ is the critical value of $g_{s i}$ for reexcitation with a discontinuous jump in $g_{\text {to }}$. Thus, the gradient imposes a logarithmic correction to the critical value of $g_{s i}$. Interestingly, this fit suggests that even for very small gradients, there is always a value of $g_{s i}$ that gives rise to reexcitation.

\section{Analysis of the slow calcium pulse}

Since reexcitation occurs during the phase 2 of the action potential, the responsible of the front diffusing to the lossdome region must be the slow inward (calcium) current $I_{s i}$. In fact, by increasing the parameter $g_{s i}$, one observes a range of values for which a slow pulse propagates, and produces reexcitations periodically, as it is shown in Fig. 6(a). To further confirm that the slow pulse observed in Fig. 6 is indeed due solely to $I_{s i}$, we run a simulation [Fig. 6(b)] in which we 
a)

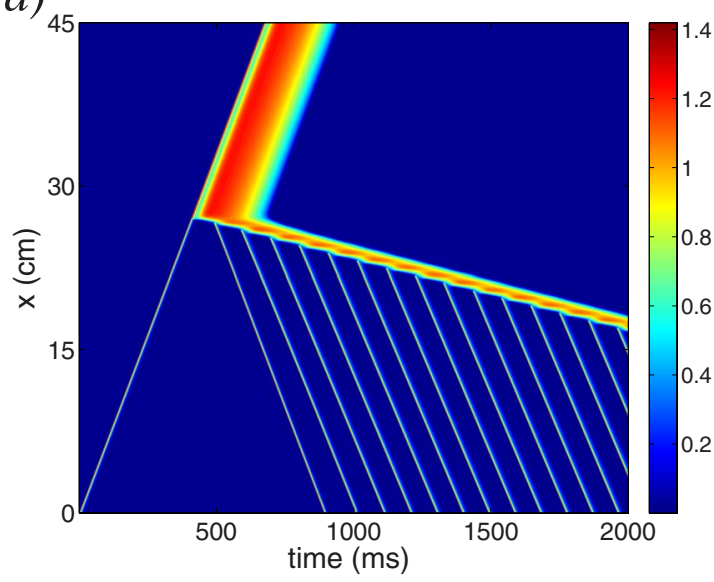

b)

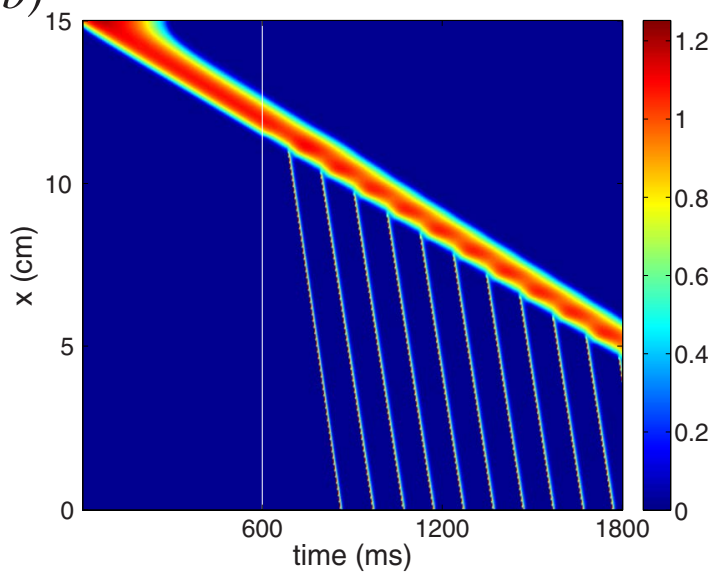

FIG. 6. (Color online) (a) Pulse with multiple BR, obtained with the parameters $g_{s i}=1.07 \times g_{s i}^{W T}, \tau_{h_{-}}=\tau_{h}^{W T} / 1.5$, and the rest of parameters are those given in Table I of Appendix A. The gradient in $g_{t o}$ is centered at $x_{d}=27 \mathrm{~cm}$ with $g_{\text {to }}^{\max }=6.2 \mathrm{~ms}^{-1}$ and $g_{\text {to }}^{\min }=3.5 \mathrm{~ms}^{-1}$. In (b), simulation with $I_{f i}$ and $I_{t o}$ initially turned off. The system size is $L=15 \mathrm{~cm}$ and $g_{t o}=6.2 \mathrm{~ms}^{-1}, g_{s i}=1.07 \times g_{s i}^{W T}, \tau_{h}=\tau_{h}^{W T} / 1.5$ in the whole domain. After an initial excitation (at time $t=0$ ), one sees the propagation of a slow pulse due solely to the slow inward current $I_{s i}$. At time $t=600 \mathrm{~ms}$, indicated by the vertical line, the currents $I_{f i}$ and $I_{t o}$ are turned on, after which periodic reexcitations appear. Note that the slow pulse remains practically unperturbed for $t>600 \mathrm{~ms}$.

set $I_{f i}$ and $I_{t o}$ equal to zero for some time $(t<600 \mathrm{~ms})$, after which we turn on all the currents $(t>600 \mathrm{~ms})$. The slow pulse is unperturbed for $t>600 \mathrm{~ms}$ but fast pulses are periodically emitted from it.

Interestingly enough, for $I_{f i}=I_{t o}=0$, the shape and the speed of the slow pulse can be calculated analytically (see the detail of these calculations in the Appendix B). Using Eqs. (B17) and (B18) we show that, as a function of the strength of the calcium conductance $g_{s i}$, the slow pulse appears trough a saddle-node bifurcation which is typical in excitable media [see Fig. 7(a)]. The position of the saddlenode bifurcation depends on the parameter $f_{0}$, which physiologically corresponds to the level at which the gate of calcium has recovered after the emission of a fast pulse. When $I_{f i}$ and $I_{t o}$ are turned on, the pulse shape is not exactly time invariant and rather oscillates with the same frequency as the frequency of reexcitations. This kind of structure is known as a "traveling breather" in the dynamicist community [32]. To further check that, in fact, this slow pulse still corresponds to the $I_{s i}$ induced pulse calculated in Appendix B, we have compared the shape of the pulses in Fig. 6(b) for $g_{s i}=1.15$ $\times g_{s i}^{W T}$. All in all, the comparison between the analytical calculations and the numerical calculations is quite good. In the cases, as in Figs. 4(c) or 4(d) where the pulse exists only transiently, one must be seeing a saddle-node remnant that attracts the solution for some time [33]. Although the determination of this time is important in order to calculate how far the transient pulse can propagate, and therefore, what is the likelihood of obtaining a reexcitation, such analytical calculation goes beyond the scope of the present paper. a)

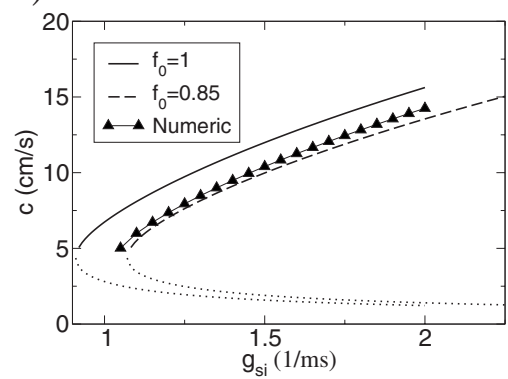

b)

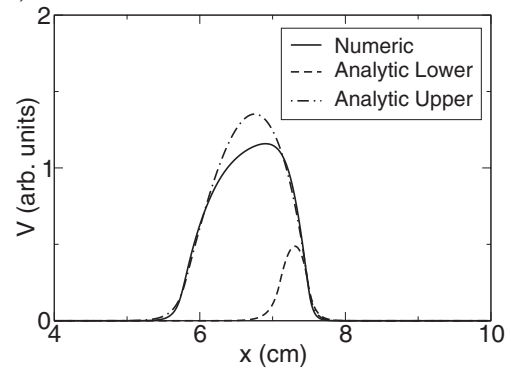

FIG. 7. (a) Velocity of the slow pulse as a function of the slow inward current conductance $g_{s i}$. Note that here $g_{s i}$ stands for $g_{s i} \times g_{s i}^{W T}$ and the rest of parameters are those given in Table I of Appendix A. These curves are obtained from the analytical theory (see Appendix B) by solving Eqs. (B17) and (B18). For each value of $g_{s i}$ there are two possible values of $c_{s i}$ corresponding to a stable (high $c_{s i}$ ) and to an unstable (low $c_{s i}$ ) branch. The slow pulse solution disappears through a saddle-node bifurcation as $g_{s i}$ is decreased below the critical value $\left(g_{s i}\right.$ $\approx 0.91$ ). The speed $c_{s i}$ depends also on the state of recovery of the gate $f$ (inactivation gate of $I_{s i}$ ) on the tissue ahead the slow pulse. For comparison sake, the triangles correspond to the velocity obtained from numerical simulations where none of the simplifying assumptions of the analytical theory are taken (see Appendix B). In (b), we compare the analytical (lower and upper branch) and the numerical slow pulse profile for parameter $g_{s i}=1.15 \times g_{s i}^{W T}$. The agreement for the shape is not as good as the agreement with the velocity due to some of the simplifying assumptions of the theory. 

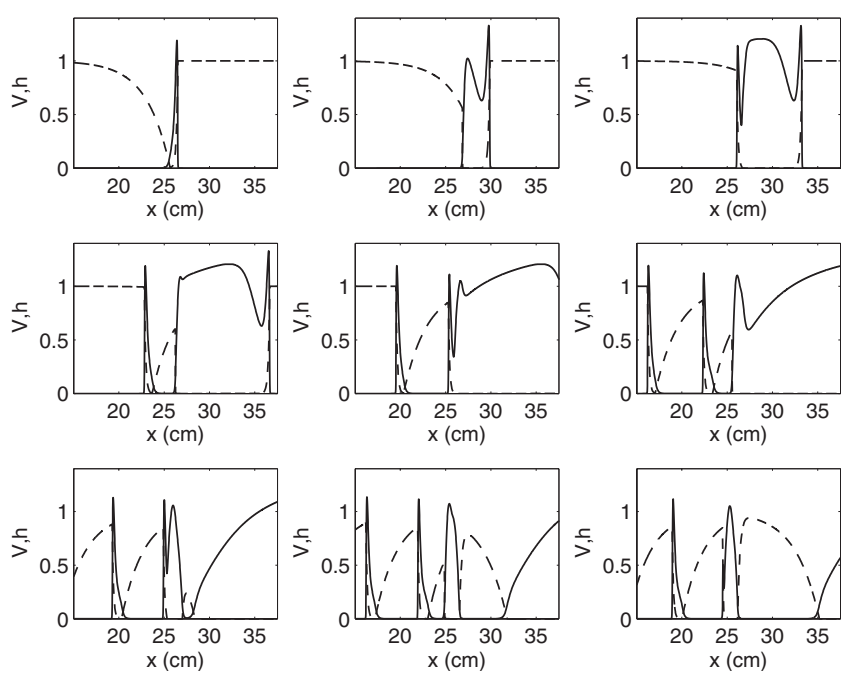

FIG. 8. Spatial distribution of voltage (solid line) and gate $h$ (dashed line), at different times, corresponding to the simulation shown in Fig. 6(a). The time difference between successive frames is $50 \mathrm{~ms}$.

\section{Kinematic description of the reexcitation phenomenon}

To obtain a better understanding of the reexcitation, we reproduce in Fig. 8 the same simulation as in Fig. 6, but showing several snapshots of the spatial distribution of voltage and the gate $h$ related to the $I_{f i}$ inactivation current. We confirm that $I_{f i}$ is not triggered exactly at the point of the fiber, $x_{d}$, where the maximum gradient of $g_{\text {to }}$ is located. At that point, rather, the recovery of the dome in the region with lower $g_{\text {to }}$ produces a gradient of transmembrane voltage that activates $I_{s i}$ in the region $\left(x<x_{d}\right)$. Spatially, this results in a retrograde front in voltage that propagates slowly into the region of high $g_{t o}\left(x<x_{d}\right)$. The slow propagation of this retrograde front is accompanied by the periodic emission of fast pulses (see the thin pulses propagating towards $x=0$ in Fig. $6)$. The exact timing of the emission of the fast pulse from the slow pulse is given by the recovery of the gate variable $h$ over a certain threshold value $\left(h>h_{*}\right)$. Thus, to obtain a reexcitation, the following condition must be fulfilled: that the system sustains a calcium $I_{s i}$ induced pulse, sufficiently long-lived to reach a point in the tissue where the fast (sodium) current has had enough time to recover and therefore produces a new fast pulse.

For the case where the slow pulse is stable the emission of the fast pulse is periodic. By using a kinematic argument, we can evaluate the time $t_{\text {reex }}$ (or distance $x_{\text {reex }}$ ) between two consecutive fast reexcitations. During the time $t_{\text {reex }}$, the slow pulse has traveled a distance $x_{\text {reex }}=t_{\text {reex }} c_{s i}$, at the speed $c_{s i}$. The fast pulse, in turn, needs a time $t_{f i}=x_{\text {reex }} / c_{f i}$ to travel the same distance $x_{\text {reex }}$ [see Fig. 9(a)]. The total time between emission will thus be $t_{\text {reex }}=t_{f i}+T$, where $T$ is the time between two consecutive reexcitations at a given fixed point in space (see Fig. 9(b)). Then, we have:

$$
T=x_{\text {reex }}\left(\frac{1}{c_{s i}}-\frac{1}{c_{f i}}\right)=\mathrm{DI}+\mathrm{APD}_{s},
$$

where $\mathrm{APD}_{s}$ is the duration of the short APD of the fast pulse that is emitted in the region of high $g_{t o}$. The velocity of the fast pulse, $c_{f i}$, can be estimated following a simplified calculation similar to the one in Ref. [36], resulting in a velocity $c_{f i}$ about one order of magnitude larger than $c_{s i}$. During the time interval $T$ the fast inward current gate has decreased, due to the emission of the short sodium pulse, and then increased again, during the recovery time DI [see Fig. 9(b)]. Therefore, we have for the inactivation gate $h$,

$$
h_{1}=1-\left(1-h_{0} e^{\left.-\mathrm{APD}_{s} / \tau_{h_{-}}\right)} e^{-\mathrm{DI} / \tau_{h_{+}}} .\right.
$$

Assuming that $h_{1}=h_{0}=h_{*}$ is the critical value of $h$ necessary to initiate a fast inward (sodium) induced reexcitation, then we have

$$
\mathrm{DI}=\tau_{h_{+}} \ln \frac{1-h_{*} e^{-\mathrm{APD}_{s} / \tau_{h_{-}}}}{1-h_{*}} .
$$

For the values of the parameters that we are considering $e^{-\mathrm{APD}_{s} / \tau_{h_{-}}} \simeq 0$, and we safely approximate DI $\simeq-\tau_{h_{+}} \ln \left(1-h_{*}\right)$. Therefore, the distance between reexcitations is given by:

$$
x_{\text {reex }} \simeq \frac{c_{s i}}{1-c_{s i} / c_{f i}}\left[\mathrm{APD}_{s}-\tau_{h_{+}} \ln \left(1-h_{*}\right)\right] .
$$

Note that this is also the minimal size that the tissue must have in order to produce a reexcitation, provided that it is orthodromic (or forward excitation FR). For typical values displayed in Fig. 9, $\mathrm{APD}_{s}=16.5 \mathrm{~ms}, h_{*}=0.83$, Eq. (6) gives $x_{\text {reex }} \simeq 0.6 \mathrm{~cm}$. In the case of a backward reexcitation (BR), the minimal distance necessary to observe a reexcitation is smaller than the typical distance between reexcitations, because the first retrograde pulse will encounter tissue that has had more time to recover (see Fig. 6). A simple argument similar to the one presented before shows that the distance $x_{\text {ref }}^{1}$ at which the first reflection is obtained is given by
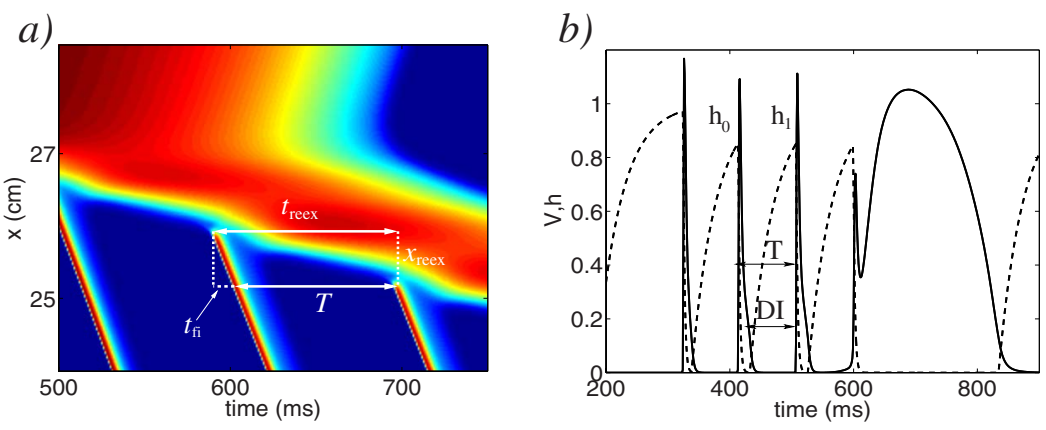

FIG. 9. (Color online) (a) Definition of the different times and distances used in the text. (b) Time evolution of the voltage and the sodium inactivation gate $h$ during several reexcitations, for the simulation displayed in Fig. 6 (cut at $\mathrm{x}=25.5 \mathrm{~cm}$ ). 

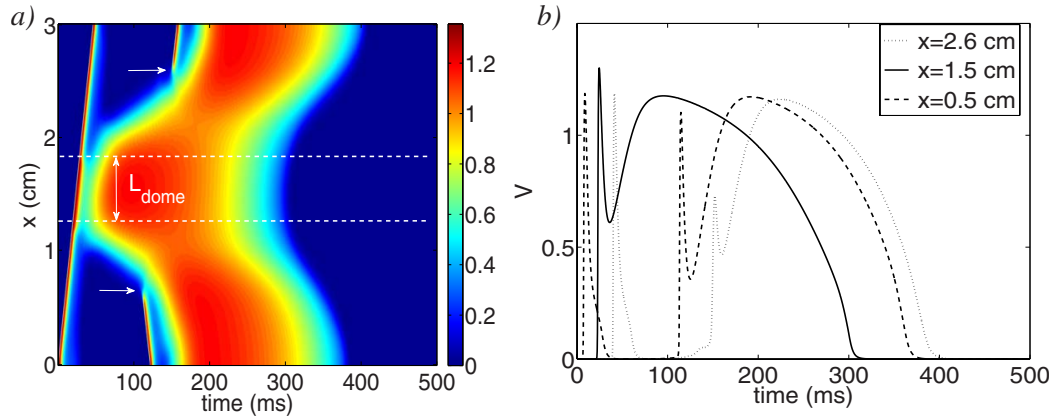

FIG. 10. (Color online) (a) Simulation of AP propagation in a cable with heterogeneous distribution of $g_{t o}$. The cable is stimulated at $x=0$ at time $t=0$. In the central region, delimited by the two Boltzmann gradients of Eq. (2), $g_{t o}$ is set to $g_{t o}^{\min }=3.5 \mathrm{~ms}^{-1}$, and in the rest of the cable $g_{t o}^{\max }=5.5 \mathrm{~ms}^{-1}$. The other parameters are those given in Table I, except that $\tau_{h_{-}}=\tau_{h_{-}}^{W T} / 1.5$, and $g_{s i}=1.07 \times g_{s i}^{W T}$. The slow pulse propagates from the spike-and-dome central region to the periphery "domeless" area, resulting in backward reexcitation (BR, indicated by the lower arrow) and forward reexcitation (FR, indicated by the upper arrow). In (b), the time evolution of the membrane potential is shown at several spatial locations.

$$
x_{r e f}^{1} \simeq \frac{c_{s i}}{1+c_{s i} / c_{f i}}\left[\mathrm{APD}_{s}-\tau_{h_{+}} \ln \left(1-h_{*}\right)\right] .
$$

which is different from the general expression for the distance between reexcitations in Eq. (6).

\section{Numerical estimates for the minimal size for reexcitation}

The expressions provided in the previous section only give a first approximation of the minimum system size needed to induce reexcitations. Furthermore, in general, the slow pulse is not stable and we have to rely on numerical simulations in order to determine this minimum system size. For that, we numerically study a one-dimensional system (a fiber) with a large value of $g_{t o}$, so the AP loses the dome, except in a central region where a lower value of $g_{t o}$ is set in order that the dome is recovered there (see Fig. 10). The fiber is paced at one end at a constant frequency of $1 \mathrm{~Hz}$. By varying the extension of the central region we are able to determine the minimal size of the spike-and-dome region necessary to obtain a reexcitation [see Fig. 11(a)] or a reflec- tion [see Fig. 11(b)]. The main result is that the smaller is the value of $g_{s i}$, the larger is the extension of the central region needed to produce reexcitation. Another result is that below some critical value of the $g_{s i}$ parameter, no reexcitation can be obtained. Interestingly enough, for high values of $g_{s i}$, a quite small central region is enough to produce reexcitations [see Figs. 11(a) and 11(b)]. In the second part of the numerical experiment, we take the minimum central region determined in the first part and measure the distance at which the FR and BR take place further apart from the central region. This second part provides the information about the extension of the region adjacent to the central region (with large $g_{t o}$ ) needed in order to get BR or FR [see Fig. 11(c) and 11(d)]. The total size of the tissue needed for the mechanism of BR or FR would therefore be the sum of the former two distances determined in part one and part two of the above numerical experiment (i.e., the central region plus the distance from the edge of the central region to the point where the fast pulse is actually emitted). As expected, there exists a value of $g_{s i}$ below which reexcitations do not occur. Clearly, a)

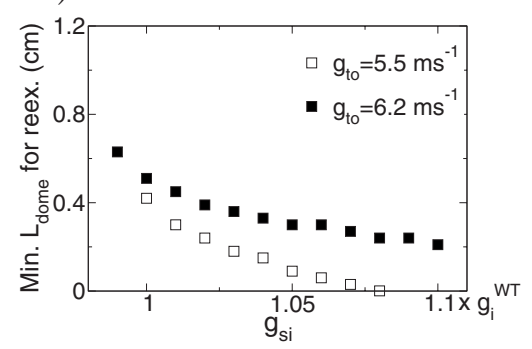

c)

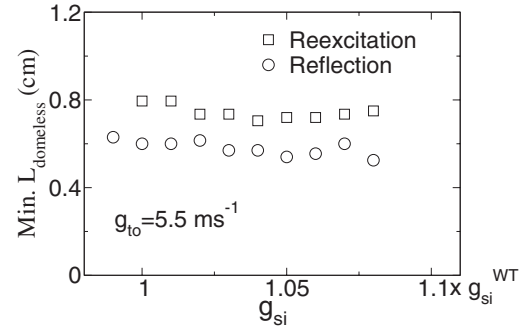

b)

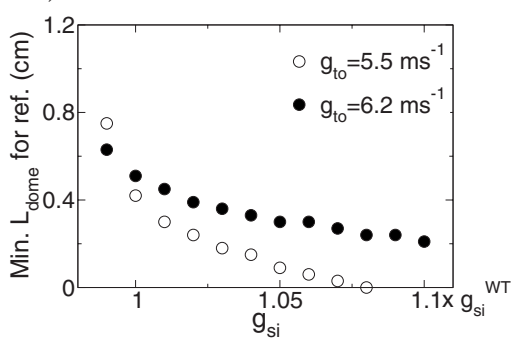

d)

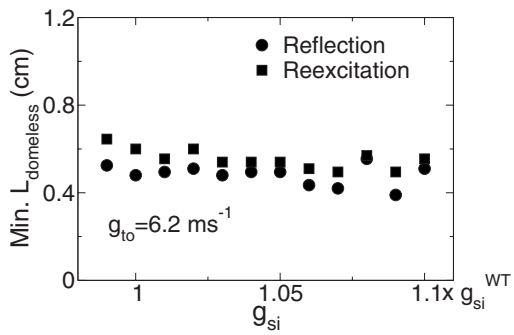

FIG. 11. Minimal size of the central region of Fig. 10 with $g_{\text {to }}^{\text {min }}=3.5 \mathrm{~ms}^{-1}$ necessary to obtain a (a) forward or (b) backward reexcitation, for a pacing frequency of $1 \mathrm{~Hz}$. For this minimal size, the minimal distance at which a FR or a BR is produced is plotted in (c) for $g_{\text {to }}^{\max }=5.5 \mathrm{~ms}^{-1}$, and in (d) for $g_{\text {to }}^{\max }=6.2 \mathrm{~ms}^{-1}$. 
the reason is that the slow pulse goes unstable before reaching regions where the fast inward current gate has had enough time to recover. Furthermore, as explained above, the minimal critical size for FR is slightly larger than for BR [compare Eqs. (6) and (7)].

\section{Three-dimensional simulations in the heart ventricles and ECG calculations}

Reexcitations produce extrasystoles that, in two and three dimensions can give rise to reentry and the generation of rotors. The occurrence of those will also depend on the geometrical arrangement of the dome and dome-less regions, this is, how the dispersion of repolarization is geometrically distributed. Let us now illustrate in a computer model of the rabbit heart ventricles the possibility of reexcitation in threedimensional realistic geometry. The details of the numerical scheme can be found in the paper by Bragard et al. [37]. The geometry of the ventricles is the one for the rabbit heart [26], including the anisotropy of the conductivity tensor at every point of the numerical domain. The numerical grid is composed by cubic voxels of $0.02 \mathrm{~cm}$ size and the time step of the explicit scheme is set to $d t=0.02 \mathrm{~ms}$. In addition to the geometry and the fiber anisotropy, one also has to include the stimulation of the AP through Purkinje fibers [38] into the numerical model. These fibers are located in the interventricular septum. In the simulations, we will present the reconstructed ECG that results from the time sequence of the depolarization of the ventricular tissue. Here, the ECG has been reconstructed using the heart dipole technique [39]. This method consists in adding all the microscopic dipoles (created at the depolarization fronts) into a single vector which is called the heart dipole vector. The next step is to project the heart vector on some standard directions in order to compute the different standard derivations of the ECG.

Previous physiological studies have shown that the presence of a more prominent $I_{t o}$ in male and/or upper right ventricular (RV) epicardium may be origin of the Brugada phenotype $[40,41]$. Following these observations, we have set a linear gradient of $g_{t o}$ in the heart ventricles. In order to do so, we set the $g_{\text {to }}$ parameter at its maximum value at a reference point in the upper ventricle with coordinates $(124,64,120)$ and the $g_{t o}$ parameter in the rest of the heart is linearly lowered by a value proportional to the distance to this reference point (see Fig. 12). We have performed three simulations with $g_{\text {to }}$ parameter in the range: (a) $g_{\text {to }} \in[3.5-4.5] \mathrm{ms}^{-1} ; \quad$ (b) $g_{\text {to }} \in[3.5-5.5] \mathrm{ms}^{-1} ;$ (c) $g_{\text {to }}$ $\in[3.5-6.5] \mathrm{ms}^{-1}$. The values of other parameters, $g_{s i}$ $=1.08 \times g_{s i}^{W T}$ and $\tau_{h}=\tau_{h}^{W T} / 1.5$, were selected in order to favor the occurrence of reexcitations.

For the three simulations, the total time is $400 \mathrm{~ms}$. In each case, only one excitation through the Purkinje fibers is sent to the ventricles at time $t=0$. Only in the "severe" situation [referred to (c) case], one observes re-entry and emergence of a disorganized electric state as shown in Fig. 13. Hence showing that a large enough gradient of $g_{t o}$ is sufficient to induce reexcitation in the same fashion as the reexcitation described in the one-dimensional simulation of a paced cable with $g_{\text {to }}$ gradients. In the other two situations where the

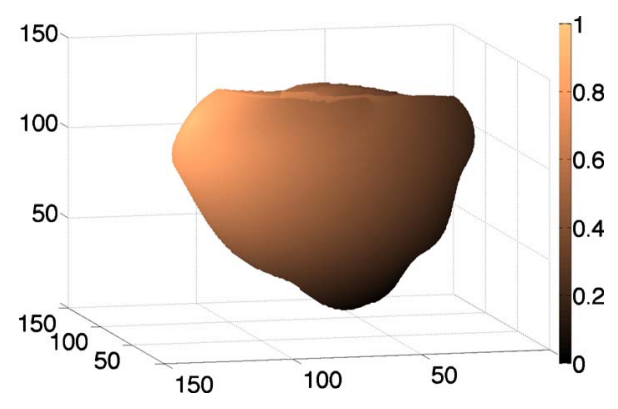

FIG. 12. (Color online) Structure of the two ventricles where the three-dimensional (3D) simulations were performed. The color scale (online) shows the normalized linear gradient of $g_{\text {to }}$ from the minimum value $(0)$ to its maximal value (1) that is reached at the reference point of coordinates $(124,64,120)$.

gradients of $g_{\text {to }}$ were lower, the initial excitation is followed by a depolarization and then successive repolarization of the entire ventricles without any reexcitation. This electrical organized activity is well captured in the corresponding ECGs shown in Fig. 14. As indicated previously, these ECGs are calculated using the approximation of the heart vector dipole in a spherical geometry for the torso, following Ref. [39].

\section{DISCUSSION}

Phase- 2 reentry is a basic mechanism for the transition to VT and VF in the heart. In this respect, a good understanding of the relevant ingredients that participate in its appearance may contribute to the development of treatments to prevent it. As discussed in the paper, reexcitation due to phase-2 reentry needs two conditions to be met: first, that a heterogeneous loss of dome is produced in tissue and, second, that the spike-and-dome regions are able to reexcitate the lossdome areas. The complication with respect to treatment is that, conditions that diminish the probability of losing the dome may also increase the probability of reexcitation. For instance, drugs that increase the strength of $I_{\mathrm{CaL}}$ may eventually help recovering the dome, and decrease dispersion of repolarization, but until this occurs, they increase the probability that a reexcitation occurs (see, for instance, Fig. 7 in Ref. [22]). This happens because an increase in $g_{\mathrm{CaL}}$ stabilizes the slow calcium pulse (see Fig. 7), that then is able to reexcitate adjacent tissue. Thus, drugs that decrease $I_{t o}$ would seem more suitable candidates to avoid dispersion of repolarization while maintaining a low probability of reexcitation, as they do not change (much) the stability of the slow pulse.

Two more results of our calculations are worth mentioning:

(i) Provided the system is in a state close to the loss of dome, very small changes, or gradients, of electrophysiological properties are enough to result in big dispersion of repolarization. Increasing $I_{t o}$ (for instance, with an activator, as in Ref. [18]), there will always be a critical value at which strong dispersion of repolarization within the epicardium will occur, since small gradients of $g_{\text {to }}$ are always present. Thus, 

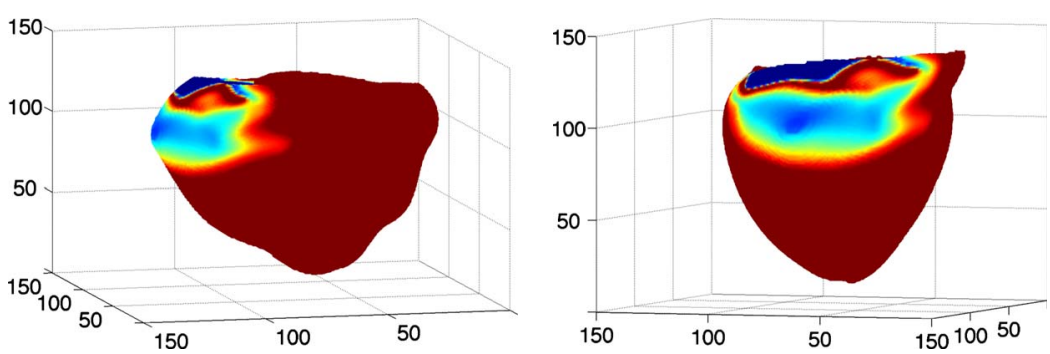

FIG. 13. (Color online) Two different viewpoints of the membrane potential $V$ distribution taken at time $t=120 \mathrm{~ms}$ after the simulation starts in the case of parameter $g_{\text {to }}$ $\in[3.5-6.5] \mathrm{ms}^{-1}$. Re-entrant activity is clearly visible in the upper RV due to the contrast of the $I_{t o}$ between the RVup region and the rest of the heart. a small change in one parameter can result in a large dispersion of repolarization in tissue, which in turn can give rise to reexcitations and, eventually, reentry.

(ii) The minimum tissue size necessary for reexcitation may be very small. Depending on parameters, sizes of the order of $0.5-1 \mathrm{~cm}$ could be enough for reexcitation (Fig. 11). Thus, the original reexcitation giving rise to VT (or VF) could occur transmurally, although dispersion of repolarization in the epicardium is necessary to allow the subsequent reentry, since the transmural wall thickness is not enough to maintain a spiral.

\section{CONCLUSIONS}

In this paper we have used a simplified five-variable model of the action potential to study the occurrence of reexcitations in a tissue with dispersion of repolarization. We have focused on the case where it appears as a result of a heterogeneous distribution of fast outward current $I_{t o}$ (together with modified sodium recovery kinetics), but similar results would be obtained if this heterogeneity was created by a variation of other suitable electrophysiological parameter, as for instance the fast inward (sodium) inactivation time. The origin of reexcitation is based on the existence (often transiently) of a slow pulse that propagates into the region of short APs until it reaches excitable tissue. In more realistic models this slow pulse is maintained by an influx of calcium through L-type calcium channels [21,22]. One interesting thing to notice is that the region of APs with dome can

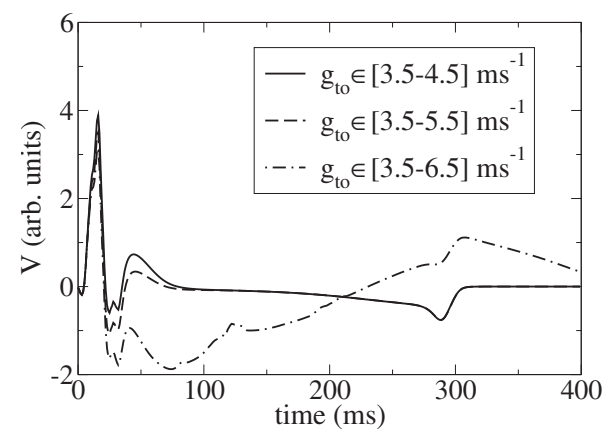

FIG. 14. Computed ECGs from the 3D simulations for the parameters $g_{s i}=1.08 \times g_{s i}^{W T}, \tau_{h}=\tau_{h}^{W T} / 1.5$, and $g_{t o}$ varying linearly in the following ranges: (a) $g_{t o} \in[3.5-4.5] \mathrm{ms}^{-1}$ (solid line); (b) $g_{\text {to }}$ $\in[3.5-5.5] \mathrm{ms}^{-1}$ (dashed line); (c) $g_{t o} \in[3.5-6.5] \mathrm{ms}^{-1}$ (dotdashed line). The lead shown here is the standard ECG first lead I [42]. Note that at time $t=120 \mathrm{~ms}$, one clearly distinguishes the reexcitation shown in Fig. 12 in the corresponding ECG (dotdashed line). be very small, and still give rise to reexcitations. For this simplified model we have obtained an estimate of the minimal tissue size necessary to obtain a reexcitation, or a reflection, which is crucially dependent on the $g_{s i}$ parameter (see Fig. 11).

Finally, we would like to emphasize the utility of considering simplified descriptions of the cardiac dynamics that, although they cannot provide complete description of the molecular mechanisms involved in the origin of channelopaties, they provide very valuable tools to study wave dynamics, and the origin of wave instabilities.

\section{ACKNOWLEDGMENTS}

This work has been financially supported by MICINN under Project Nos. FIS2008-06335-C02-01, FIS2008-06335C02-02, and FIS2009-13360-C03-03.

\section{APPENDIX A: DEFINITION AND PARAMETER VALUES FOR THE FIVE-VARIABLE MODEL}

In dimensionless form the cable equation is

$$
\frac{\partial V}{\partial t}=\nabla \cdot(\mathbf{D} \nabla V)-I_{\text {ion }},
$$

with

$$
I_{i o n}=I_{f i}+I_{s i}+I_{s o}+I_{t o}
$$

and

$$
\begin{gathered}
I_{f i}=-g_{f i} h m_{\infty}\left(V_{f i}-V\right), \\
I_{s i}=-g_{s i} d_{\infty} f f_{\infty}^{\prime}, \\
I_{t o}=g_{t o} r s V, \\
I_{s o}=g_{s o} k_{\infty} .
\end{gathered}
$$

The gates follow standard kinetics

$$
\begin{gathered}
\dot{h}=\left(h_{\infty}(V)-h\right) / \tau_{h}(V), \\
\dot{f}=\left(f_{\infty}(V)-f\right) / \tau_{f}(V), \\
\dot{r}=\left(r_{\infty}(V)-r\right) / \tau_{r}(V), \\
\dot{s}=\left(s_{\infty}(V)-s\right) / \tau_{s}(V),
\end{gathered}
$$

with the steady-state values of the gates and the time constants given by 


$$
\begin{gathered}
m_{\infty}=\left(V-V_{c}\right) \Theta\left(V-V_{c}\right), \\
h_{\infty}=f_{\infty}=s_{\infty}=\Theta\left(V_{c}-V\right), \\
d_{\infty}=\Theta\left(V-V_{c}\right)\left(1+\tanh \left[\beta_{1}\left(V-V_{1}\right)\right]\right) / 2, \\
f_{\infty}^{\prime}=\left(1-\tanh \left[\beta_{2}\left(V-V_{2}\right)\right]\right) / 2, \\
k_{\infty}=\Theta\left(V-V_{c}\right)+V / V_{c} \Theta\left(V_{c}-V\right), \\
r_{\infty}=\Theta\left(V-V_{r}\right), \\
\tau_{h}=\tau_{h_{-}}+\left(\tau_{h_{+}}-\tau_{h_{-}}\right) \Theta\left(V_{c}-V\right), \\
\tau_{f}=\tau_{f_{-}}+\left(\tau_{f_{+}}-\tau_{f_{-}}\right) \Theta\left(V_{c}-V\right), \\
\tau_{r}=\tau_{r_{-}}+\left(\tau_{r_{+}}-\tau_{r_{-}}\right) \Theta\left(V-V_{r}\right), \\
\tau_{s}=\tau_{s_{-}}+\left(\tau_{s_{+}}-\tau_{s_{-}}\right) \Theta\left(V_{c}-V\right),
\end{gathered}
$$

The values of the different parameters of the model are given in Table I and they correspond to the experimental epicardial action potential described in Ref. [29].

\section{APPENDIX B: EXISTENCE AND PROPERTIES OF THE SLOW CALCIUM PULSE}

The analytical analysis starts with the one-dimensional cable equation in dimensionless form

$$
\frac{\partial V}{\partial t}=\nabla(\mathbf{D} \nabla V)-I_{i o n},
$$

where we assume that the currents $I_{f i}$ and $I_{t o}$, responsible for phases 0 and 1 of the AP, do not contribute to the existence and propagation of the slow pulse. To simplify the calculation we will also assume that $\left(1+\tanh \left[\beta_{1}\left(V-V_{1}\right)\right]\right) / 2$ $\simeq \Theta\left(V-V_{1}\right)$, and that $\left(1-\tanh \left[\beta_{2}\left(V-V_{2}\right)\right]\right) / 2 \simeq \Theta\left(V_{2}-V\right)$. Although it is not necessary, to even further simplify the calculations, we will also assume that the threshold for the slow outward current is the same as the threshold for the opening of $I_{s i}$ (i.e., that $V_{c}=V_{1}$ ). Then, the equation for the transmembrane voltage can be expressed as

$$
\begin{aligned}
\frac{\partial V}{\partial t}= & D \frac{\partial^{2} V}{\partial x^{2}}+g_{s i} f(t) \Theta\left(V-V_{1}\right) \Theta\left(V_{2}-V\right) \\
& -g_{s o} \Theta\left(V-V_{1}\right)-g_{s o} \frac{V}{V_{1}} \Theta\left(V_{1}-V\right)
\end{aligned}
$$

or by separating Eq. (B2) according to the values of the voltage

$$
\begin{gathered}
\frac{\partial V}{\partial t}=D \frac{\partial^{2} V}{\partial x^{2}}-g_{s o} \frac{V}{V_{1}} \quad V<V_{1}, \\
\frac{\partial V}{\partial t}=D \frac{\partial^{2} V}{\partial x^{2}}+g_{s i} f(t)-g_{s o} \quad V_{1} \leq V \leq V_{2},
\end{gathered}
$$

$$
\frac{\partial V}{\partial t}=D \frac{\partial^{2} V}{\partial x^{2}}-g_{s o} \quad V>V_{2} .
$$

A last simplification done in the present analysis is to solve only Eqs. (B3) and (B4) and not Eq. (B5). As can be seen in Figs. 7(a) and 7(b), this last simplification will not affect the pulse velocity but rather affect the pulse shape (especially far from the bifurcation). A better comparison of the pulse shape would need to take also into account Eq. (B5) in the calculations but then the algebra becomes rather cumbersome.

Let us assume that the calcium pulse is moving from left to right with a constant velocity of value $c_{s i}$, and let us work in the moving frame coordinate $\xi=x-c_{s i} t$. Using this change of variable, we then separate the space $(\xi)$ into three regions according to the values of the transmembrane voltage, i.e., region I, from $\xi=0$ to $\xi=+\infty$; region II, from $\xi=-\xi_{0}$ to $\xi$ $=0$; and region III from $\xi=-\infty$ to $\xi=-\xi_{0}$. Let us fix $\xi=-\xi_{0}$ and $\xi=0$, the positions where the transmembrane voltage takes the value $V(0)=V\left(-\xi_{0}\right)=V_{1}$.

Equation (B3), which is valid for regions I and III, can be expressed as a function of the variable $\xi$ in the moving frame as

$$
-c_{s i} \frac{\partial V}{\partial \xi}=D \frac{\partial^{2} V}{\partial \xi^{2}}-g_{s o} \frac{V}{V_{1}},
$$

with the boundary conditions $V=0$ at $\xi=+\infty$ and $\xi=-\infty$.

For region II, Eq. (B4) must be solved,

$$
-c_{s i} \frac{\partial V}{\partial \xi}=D \frac{\partial^{2} V}{\partial \xi^{2}}+g_{s i} f(\xi)-g_{s o},
$$

where

$$
f(\xi)=f_{0}(V) e^{\xi / c_{s i} \tau_{f}}
$$

and $\tau_{f}$ takes the value $\tau_{f}$. By solving the above ordinary differential equations in the three regions and by imposing the corresponding boundary conditions, we readily obtain the following expressions for the transmembrane voltage:

$$
V(\xi)=V_{1} e^{\lambda_{2} \xi} \quad \text { Region I, }
$$

$V(\xi)=A_{3}+A_{4} e^{-c_{s i} \xi / D}+\frac{g_{s o}}{c_{s i}} \xi-\frac{g_{s i} f_{0} \tau_{f}}{1+D / c_{s i}^{2} \tau_{f}} e^{\xi / c_{s i} \tau_{f}}$ Region II,

$$
V(\xi)=V_{1} e^{\lambda_{1}\left(\xi+\xi_{0}\right)} \quad \text { Region III, }
$$

with the spatial growth rates

$$
\begin{aligned}
& \lambda_{2}=\frac{c_{s i}}{2 D}\left[-1+\sqrt{1+\frac{4 D g_{s o}}{c_{s i}^{2} V_{1}}}\right]>0, \\
& \lambda_{1}=\frac{c_{s i}}{2 D}\left[-1-\sqrt{1+\frac{4 D g_{s o}}{c_{s i}^{2} V_{1}}}\right]<0 .
\end{aligned}
$$

The remaining constants $A_{3}$ and $A_{4}$ are determined by matching the voltage and its first derivative between region I and region II at $\xi=0$. One gets 


$$
\begin{gathered}
A_{4}=\frac{D P_{2}}{c_{s i}}, \\
A_{3}=-A_{4}+V_{1}+\frac{g_{s i} f_{0} \tau_{f}}{1+D / c_{s i}^{2} \tau_{f}},
\end{gathered}
$$

where one has defined an intermediate variable $P_{2}$ in order to alleviate the notation,

$$
P_{2}=\frac{g_{s o}}{c_{s i}}-\frac{g_{s i} f_{0}}{c_{s i}\left(1+D / c_{s i}^{2} \tau_{f}\right)}-\lambda_{2} V_{1} .
$$

Then, by matching the voltage and its first derivative between regions II and III at $\xi=-\xi_{0}$, the values of the pulse speed $c_{s i}$ and the pulse width $\xi_{0}$ are determined by solving numerically the two following transcendental equations:

$$
\frac{g_{s o}}{c_{s i}}-\lambda_{1} V_{1}-e^{c_{s i} \xi_{0} / D} P_{2}-e^{-\xi_{0} / c_{s i} \tau_{f}} \frac{g_{s i} f_{0}}{c_{s i}\left(1+D / c_{s i}^{2} \tau_{f}\right)}=0,
$$

$$
\begin{gathered}
\frac{D}{c_{s i}}\left(P_{2} e^{c_{s i} \xi_{0} / D}+\lambda_{2} V_{1}\right)-\frac{g_{s o} \xi_{0}}{c_{s i}}-\frac{D g_{s o}}{c_{s i}^{2}}+g_{s i} f_{0} \tau_{f} \\
-\frac{g_{s i} f_{0} \tau_{f}}{1+D / c_{s i}^{2} \tau_{f}} e^{-\xi_{0} / c_{s i} \tau_{f}}=0 .
\end{gathered}
$$

The results of the analysis are summarized in Figs. 7(a) and 7(b). The analysis of stability of the slow pulse solution is beyond the scope of the present paper.
[1] World Health Report 2004, Statistical Annex (Table 2).

[2] C. Antzelevitch, Heart Rhythm 4, 964 (2007).

[3] J. L. Greenstein, R. Wu, S. Po, G. F. Tomaselli, and R. L. Winslow, Circ. Res. 87, 1026 (2000).

[4] M. Dong, S. Yan, Y. Chen, P. J. Niklewski, X. Sun, K. Chenault, and H.-S. Wang, J. Cardiovasc. Electrophysiol. 21, 697 (2010).

[5] J. M. Di Diego, Z. Q. Sun, and C. Antzelevitch, Am. J. Physiol. 271, H548 (1996).

[6] A. Lukas and C. Antzelevitch, Cardiovasc. Res. 32, 593 (1996).

[7] J. M. Di Diego and C. Antzelevitch, Circulation 89, 1839 (1994).

[8] P. Brugada and J. Brugada, J. Am. Coll. Cardiol. 20, 1391 (1992).

[9] Study group on molecular basis of arrhythmias. Inherited Arrhythmias Database http://www.fsm.it/cardmoc/

[10] Q. Chen, G. E. Kirsch, D. Zhang, R. Brugada, J. Brugada, P. Brugada, D. Potenza, A. Moya, M. Borggrefe, G. Breithardt, R. Ortiz-Lopez, Z. Wang, C. Antzelevitch, R. E. O'Brien, E. Schulze-Bahr, M. T. Keating, J. A. Towbin, and Q. Wang, Nature (London) 392, 293 (1998).

[11] C. Antzelevitch, G. D. Pollevick, J. M. Cordeiro, O. Casis, M. C. Sanguinetti, Y. Aizawa, A. Guerchicoff, R. Pfeiffer, A. Oliva, B. Wollnik, P. Gelber, E. P. Bonaros, E. Burashnikov, Y. Wu, J. D. Sargent, S. Schickel, R. Oberheiden, A. Bhatia, L.-F. Hsu, M. Haïssaguerre, R. Schimpf, M. Borggrefe, and C. Wolpert, Circulation 115, 442 (2007).

[12] A. A. Wilde, C. Antzelevitch, M. Borggrefe, J. Brugada, R. Brugada, P. Brugada, D. Corrado, R. N. W. Hauer, R. S. Kass, K. Nademanee, S. G. Priori, and J. A. Towbin, Circulation 106, 2514 (2002).

[13] P. B. Bennett, K. Yazawa, N. Makita, and A. L. George, Jr., Nature (London) 376, 683 (1995).

[14] C. Antzelevitch, P. Brugada, J. Brugada, and R. Brugada, The Brugada Syndrome: From Bench to Bedside (Blackwell Futura, Oxford, UK, 2005).

[15] C. Antzelevitch, Am. J. Physiol. Heart Circ. Physiol. 293, H2024 (2007).

[16] M. V. Brahmajothi, D. L. Campbell, R. L. Rasmusson, M. J.
Morales, J. S. Trimmer, J. M. Nerbonne, and C. S. Harold, J. Gen. Physiol. 113, 581 (1999).

[17] G. Y. Oudit, Z. Kassiri, R. Sah, R. J. Ramirez, C. Zobel, and P. H. Backx, J. Mol. Cell. Cardiol. 33, 851 (2001).

[18] K. Calloe, J. M. Cordeiro, J. M. Di Diego, R. S. Hansen, M. Grunnet, S. P. Olesen, and C. Antzelevitch, Cardiovasc. Res. 81, 686 (2009).

[19] E. Delpón, J. M. Cordeiro, L. Núez, P. E. B. Thomsen, A. Guerchicoff, G. D. Pollevick, Y. Wu, J. K. Kanters, C. T. Larsen, E. Burashnikov, M. Christiansen, and C. Antzelevitch, Circulation 1, 209 (2008).

[20] S. Miyoshi, H. Mitamura, K. Fujikura, Y. Fukuda, K. Tanimoto, Y. Hagiwara, M. Ita, and S. Ogawa, Am. J. Physiol. Heart Circ. Physiol. 284, H1285 (2003).

[21] A. Maoz, T. Krogh-Madsen, and D. J. Christini, Heart Rhythm 6, 813 (2009).

[22] I. R. Cantalapiedra, A. Peñaranda, L. Mont, J. Brugada, and B. Echebarria, J. Theor. Biol. 259, 850 (2009).

[23] A. Peñaranda, I. R. Cantalapiedra, J. Bragard, and B. Echebarria, Cardiac Dynamics: A Simplified Model for Action Potential Propagation, (unpublished).

[24] J. Keener and J. Sneyd, Mathematical Physiology (SpringerVerlag, New York, 2001).

[25] A. Pullan, M. Buist, and L. Cheng, Mathematically Modelling the Electrical Activity of the Heart (World Scientific Publisher, Singapore, 2005).

[26] F. J. Vetter and A. D. McCullogh, Prog. Biophys. Mol. Biol. 69, 157 (1998).

[27] F. Aguel, J. Eason, and N. Trayanova, Int. J. Bifurcat. Chaos 13, 3791 (2003).

[28] F. Fenton and A. Karma, Chaos 8, 20 (1998).

[29] M. Nabauer, D. J. Beuckelmann, P. Uberfuhr, and G. Steinbeck, Am. J. Physiol. 292, H43 (1996).

[30] Matlab7 from Mathworks Inc., Natick, MA (USA).

[31] T. Aiba, W. Shimizu, I. Hidaka, K. Uemura, T. Noda, C. Zheng, A. Kamiya, M. Inagaki, M. Sugimachi, and K. Sunagawa, J. Am. Coll. Cardiol. 47, 2074 (2006).

[32] N. Akhmediev, J. M. Soto-Crespo, and G. Town, Phys. Rev. E 63, 056602 (2001).

[33] S. Strogatz, Nonlinear Dynamics and Chaos with Applications 
to Physics, Biology, Chemistry, and Engineering (Westview, Boulder, CO, 2000).

[34] S. P. Patel and D. L. Campbell, J. Physiol. 569, 7 (2005).

[35] H. Xu, W. Guo, and J. M. Nerbonne, J. Gen. Physiol. 113, 661 (1999).

[36] J. W. Cain, E. G. Tolkacheva, D. G. Schaeffer, and D. J. Gauthier, Phys. Rev. E 70, 061906 (2004).

[37] J. Bragard, E. Cherry, N. Otani, and F. Fenton, Modelization of Action Potential through the Heart's Ventricles using Bidomain Formulation (unpublished).

[38] A. M. Katz, Physiology of the Heart (Lippincott Williams \& Wilkins, Philadelphia, 2005).
[39] Quantitative Physiology, Organ Transport Systems by Prof. Roger G. Mark and Prof. Jose Venegas (MIT), 2004, see the following Web page: http://ocw.mit.edu/OcwWeb/ Health-Sciences-and-Technology/HST-542JSpring-2004/ CourseHome/index.htm

[40] J. Di Diego, Z. Sun, and C. Antzelevitch, Am. J. Physiol. 271, 548 (1996)

[41] J. Di Diego, J. Cordeiro, R. Goodrow, J. Fish, A. Zygmunt, G. Perez, F. Scornik, and C. Antzelevitch, Circulation 106, 2004 (2002).

[42] T. B. Garcia and G. T. Miller, Arrhythmia Recognition: The Art of Interpretation (Jones \& Bartlett Publishers, Sudbury, 2004). 